\title{
Hypothermia inhibits the propagation of acute ischemic injury by inhibiting HMGB1

Jung Ho Lee ${ }^{1 \dagger}$, Eun Jang Yoon ${ }^{1 \dagger}$, Jeho Seo ${ }^{1}$, Adriana Kavoussi', Yong Eun Chung ${ }^{2}$, Sung Phil Chung ${ }^{3}$, Incheol Park ${ }^{3}$ Chul Hoon Kim ${ }^{1,4^{*}}$ and Je Sung You ${ }^{3^{*}}$

\begin{abstract}
Acute ischemic stroke causes significant chronic disability worldwide. We designed this study to clarify the mechanism by which hypothermia helps alleviate acute ischemic stroke. In a middle cerebral artery occlusion model ( $4 \mathrm{~h}$ ischemia without reperfusion), hypothermia effectively reduces mean infarct volume. Hypothermia also prevents neurons in the infarct area from releasing high mobility group box 1 (HMGB1), the most well-studied damage-associated molecular pattern protein. By preventing its release, hypothermia also prevents the typical middle cerebral artery occlusion-induced increase in serum HMGB1. We also found that both glycyrrhizin-mediated inhibition of HMGB1 and intracerebroventricular neutralizing antibody treatments before middle cerebral artery occlusion onset diminish infarct volume. This suggests a clear neuroprotective effect of HMGB1 inhibition by hypothermia in the brain. We next used real-time polymerase chain reaction to measure the levels of pro-inflammatory cytokines in peri-infarct regions. Although middle cerebral artery occlusion increases the expression of interleukin-1 $\beta$ and tissue necrosis factor-a, this elevation is suppressed by both hypothermia and glycyrrhizin treatment. We show that hypothermia reduces the production of inflammatory cytokines and helps salvage peri-infarct regions from the propagation of ischemic injury via HMGB1 blockade. In addition to suggesting a potential mechanism for hypothermia's therapeutic effects, our results suggest HMGB1 modulation may lengthen the therapeutic window for stroke treatments.
\end{abstract}

Keywords: Acute ischemic stroke, High mobility group box 1 (HMGB1), Inflammatory cytokines, Penumbra, Hypothermia, Glycyrrhizin

Abbreviations: BBB, Blood brain barrier; CCA, Common carotid artery; DAMP, Damage-associated molecular pattern; DAPI, 4,6-diamidino-2-phenylindole; ECA, External carotid artery; ELISA, Enzyme-linked immunosorbent assay;

HMGB1, High mobility group box 1; ICA, Internal carotid artery; IL-1 $\beta$, Interleukin-1 $\beta$; IV-tPA, Intravenous tissue plasminogen activator; MCAO, Middle cerebral artery occlusion; RAGE, Receptor for advanced glycation end-products; TLR4, Toll-like receptor 4; TNF-a, Tissue necrosis factor-a; TTC, 2,3,5-triphenyltetrazolium chloride

\section{Introduction}

Traditional stroke treatments comprise aspirin, surgery, and thrombolysis [1-3]. If they can be rapidly applied, intravenous tissue plasminogen activator (IV-tPA) and intra-arterial thrombolysis are the most effective treatments

\footnotetext{
*Correspondence: kimhoon@yuhs.ac; youjsmd@yuhs.ac

${ }^{\dagger}$ Equal contributors

'Department of Pharmacology, BK21 PLUS Project for Medical Science, Brain Research Institute, Yonsei University College of Medicine, 50-1 Yonsei-ro, Seodaemun-gu, Seoul 03722, South Korea

${ }^{3}$ Department of Emergency Medicine, Yonsei University College of Medicine, 50-1 Yonsei-ro, Seodaemun-gu, Seoul 03722, South Korea

Full list of author information is available at the end of the article
}

for ischemic strokes [4]. Although it has long been recognized that treatment should begin within $3 \sim 4.5 \mathrm{~h}$ of stroke onset $[3,5]$, circumstances prevent many patients from receiving the most effective treatments within the optimal time interval [6, 7]. Thus, new adjunctive treatments that extend this critical therapeutic window will be invaluable in improving outcomes for patients with ischemic brain injuries.

The ischemic penumbra is an important target for stroke therapeutics. According to its original delineation, the ischemic penumbra is the region of brain tissue receiving reduced cerebral blood flow that surrounds the 
infarct core [8]. If a therapeutic intervention fails, the ischemic penumbra can also be encompassed into the developing infarct core $[9,10]$. Therapeutic hypothermia has been suggested as a way to 'freeze' the ischemic penumbra and prolong the therapeutic window for acute ischemic stroke $[11,12]$. Therapeutic hypothermia-accomplished by cooling a patient's core body temperature to $32-34{ }^{\circ} \mathrm{C}$ for $12-24 \mathrm{~h}$-offers strong neuroprotection for survivors of cardiac arrest [13]. Although the therapeutic efficacy of hypothermia for acute ischemic stroke has not yet been established in a large-scale clinical trial, several smaller studies have successfully demonstrated feasibility $[14,15]$. In rodent models, hypothermia seems to reduce both ischemic core and penumbral injuries [16-19]. Hypothermia is known to reduce excitatory neurotransmitter release and free radical production, maintain blood brain barrier (BBB) integrity, and mitigate ischemiainduced inflammation [19-22]. Still, the principal molecular mediator of these neuroprotective effects of hypothermia is unknown.

Acute ischemic strokes trigger an innate immune response, leading to severe inflammation [23]. High mobility group box 1 (HMGB1), which is released from several types of cells upon injury, is one of the primary mediators of this innate immune response [24, 25]. HMGB1 is also a critical mediator of both the primary and secondary damage caused by ischemic strokes [24-26]. Indeed, interruption of HMGB1's role as a damage-associated molecular pattern (DAMP) protein helps prevent the propagation of ischemic injury [24]. Extracellular HMGB1 binds to toll-like receptor 4 and receptor for advanced glycation end-products expressed in immune-competent cells, neurons, and astrocytes [27, 28]. HMGB1 binding acts as a danger signal, activating inflammatory mediators that then amplify and expand the extent of brain damage [26]. For example, in glial and endothelial cell, HMGB1 induces the expression of inflammatory mediators, tissue necrosis factor (TNF)- $\alpha$ and intercellular adhesion molecule (ICAM)-1 [29]. The upregulation of metalloproteinase (MMP)- 9 by HMGB1 in neurons and astrocytes damages blood-brain barrier and expands brain damage [30]. In stroke patients, serum HMGB1 levels positively correlate with stroke severity [26, 31]. These reports suggest that HMGB1 is a valuable molecular target for new adjunctive stroke therapies.

Here we present evidence using a rat middle cerebral artery occlusion (MCAO) model of ischemic stroke that hypothermia inhibits infarct volume expansion by preventing HMGB1 release from postischemic neurons and diminishes subsequent inflammatory responses in the peri-infarct region. This study clarifies the mechanism by which hypothermia exerts its therapeutic effects and suggests that further interventions aimed at blocking the actions of
HMGB1 will be valuable additions to stroke treatment regimens.

\section{Results \\ Therapeutic hypothermia reduces infarct volume in post-ischemic brains}

In this study, we used a permanent MCAO model to explore the molecular changes induced by hypothermia prior to recanalization. We induced hypothermia $15 \mathrm{~min}$ after MCAO surgery and maintained it throughout the entire $4 \mathrm{~h}$ ischemic period (Fig. 1a). We ensured constant hypo- or normothermia by directly monitoring the core temperature of each rat. The core temperature of hypothermic rats fell to $33.0 \pm 0.5{ }^{\circ} \mathrm{C}$ within $80 \mathrm{~min}$ and remained constant for $4 \mathrm{~h}$ following MCAO (Fig. 1b). The redox indicator 2,3,5-triphenyltetrazolium chloride (TTC) effectively delineates cerebral infarct volume with infarcted areas appearing devoid of red staining (Fig. 1c, see also in Additional file 1: Figure S1). Absent or attenuated staining of MAP2, a cytoskeletal protein that is highly sensitive to ischemic damage, also delineates a similar infarct area as the lack of TTC conversion to red-colored triphenylformazane does (in Additional file 2: Figure S2). Infarcts induced by $4 \mathrm{~h} \mathrm{MCAO}$ are significantly diminished by hypothermia at $33{ }^{\circ} \mathrm{C}$ compared with normothermia (Fig. 1c, see also in Additional file 1: Figure S1). By measuring TTC staining-negative tissue volumes, we found that mild hypothermia at $33{ }^{\circ} \mathrm{C}$ attenuates mean infarct volume from $256.40 \pm 29.01 \mathrm{~mm}^{3}$ to $73.60 \pm 37.67 \mathrm{~mm}^{3}$ (Fig. 1d). MCAO-induced neurological deficits were evaluated using behavioral tests and found to be improved by hypothermia treatment (in Additional file 3: Figure S3). These data indicate hypothermia protects brain cells against cerebral ischemic damage.

\section{Therapeutic hypothermia inhibits extracellular release of HMGB1 from ischemic brain tissue}

Upon MCAO-induced ischemic injury, HMGB1 is released from brain cell nuclei, reducing the number of HMGB1-positive cells in the ischemic cortex [24, 29]. We too found that HMGB1 immunoreactivity disappears from the cortex of MCAO rats, but that hypothermia significantly restores HMGB1 staining in the post-ischemic cortex. This suggests hypothermia attenuates the extracellular release of HMGB1 (Fig. 2a). We observed that while $30.31 \pm 1.60 \%$ of 4,6-diamidino2-phenylindole (DAPI)-positive cells in the cortex of ischemic hemispheres (ipsilateral) were also HMGB1postive, hypothermia increases this number roughly two-fold to $65.04 \pm 3.53 \%$ (Fig. 2b). Next, we performed an ELISA to measure HMGB1 levels in serum samples obtained $4 \mathrm{~h}$ after the onset of ischemia. As expected, the level of circulating of HMGB1 rises after MCAO, but its rise is significantly attenuated by hypothermia 

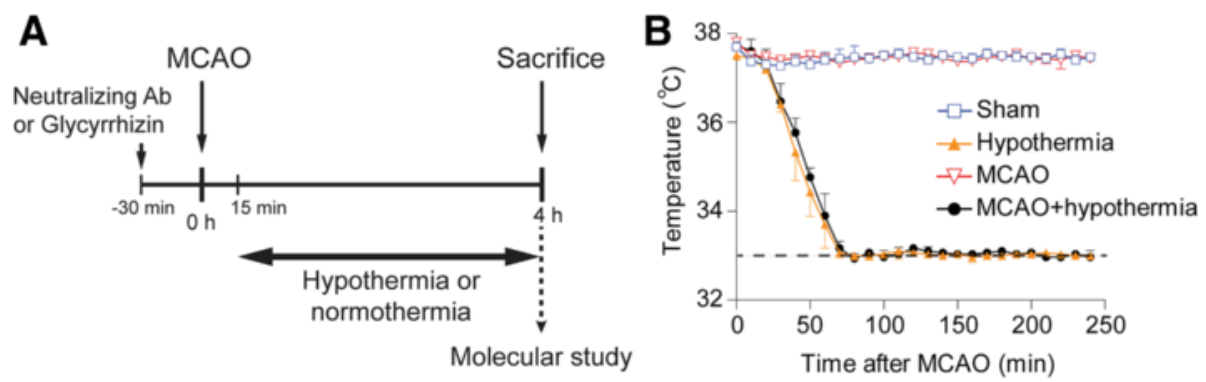

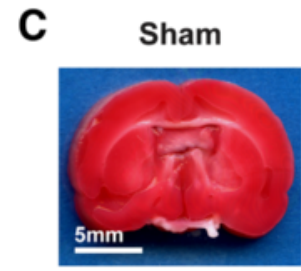

Hypothermia
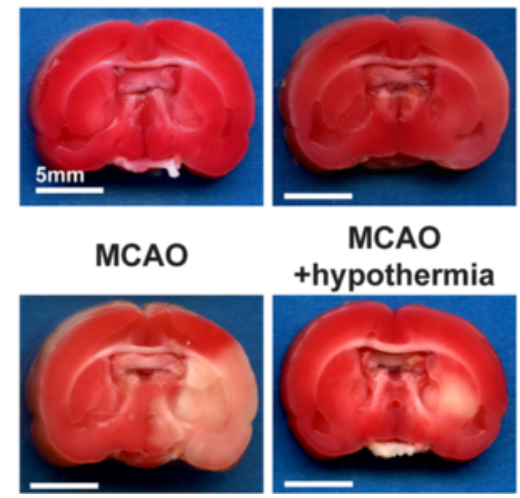

MCAO +hypothermia

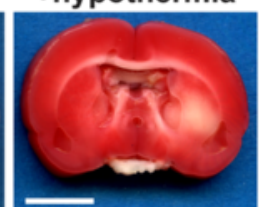

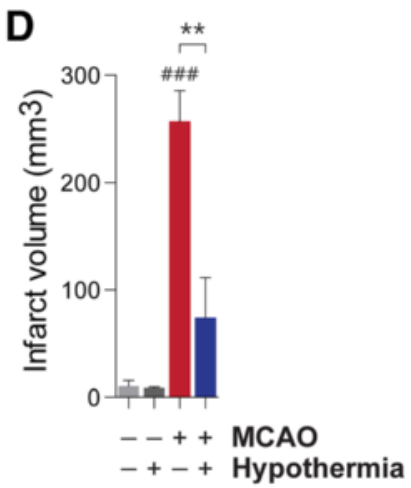

Fig. 1 Hypothermia reduces ischemic infarct volume in MCAO rats. a, An illustration of the experimental schedule. b, Body temperature traces in rats after middle cerebral artery occlusion (MCAO). c, Representative images of 2,3,5-triphenyltetrazolium chloride (TTC) staining results. $\mathbf{d}$, A quantification of infarct volume measured in TTC-stained brain slices of rats treated with MCAO and/or hypothermia. The number of rats in each group was as follows: sham $(n=3)$, hypothermia $(n=4)$, MCAO $(n=5)$, MCAO + hypothermia $(n=4)$. \#\#\# $P<0.001$ versus sham alone, ${ }^{* *} P<0.01$ comparing MCAO with and without hypothermia, one-way analysis of variance (ANOVA) followed by the Bonferroni post hoc test

(Fig. 3). To evaluate if HMGB1 rise by MCAO affects neuron directly, we examined whether HMGB1 is preferentially depleted in the neuronal marker-positive cells. We stained rat brain sections with antibodies against HMGB1 and the neuronal marker NeuN. In the ipsilateral sham group, we found that $80.02 \pm 2.27 \%$ of HMGB1-positive cells were also NeuN-positive (Fig. 4a and b). Studies using the in vivo MCAO model and an oxygen-glucose deprivation in vitro culture model both report similar selective neuronal release of HMGB1 [26, 29]. In our MCAO rats, we observed a significant drop in the percentage of NeuN/HMGB1 double-positive cells to $39.0 \pm 2.94 \%$ of HMGB1-positive cells. This reduction, too, is dramatically restored by hypothermia-treated rats show $71.98 \pm 2.72 \%$ NeuN/HMGB1-double positive cells (Fig. 4a and b). These results indicate that hypothermia blocks the release of HMGB1 from ischemic rat neurons post-MCAO.

\section{Glycyrrhizin ameliorates MCAO-induced ischemic brain injury}

Glycyrrhizin is a pharmacological HMGB1 inhibitor that has been suggested to bind directly to HMGB1 blocking its function as a cytokine [32] and to prevent cellular HMGB1 release [33, 34]. In an effort to verify that the neuroprotection conferred by hypothermia in our experimental MCAO model acts via inhibition of HMGB1, we measured the effect of glycyrrhizin treatment on infarct volume as well as HMGB1 release. Intra-peritoneal injection of glycyrrhizin markedly attenuates infarct volume in the post-ischemic cortex $\left(257.20 \pm 21.93 \mathrm{~mm}^{3}\right.$ in MCAO rats versus $77.35 \pm 27.19 \mathrm{~mm}^{3}$ in glycyrrhizintreated MCAO rats, $P<0.001$ ) (Fig. $5 \mathrm{a}$ and $\mathrm{b}$, see also in Additional file 4: Figure S4). Glycyrrhizin also significantly increases the percentage of HMGB1-positive cells in the ischemic hemisphere $(30.23 \pm 1.34 \%$ in MCAO rats versus $52.17 \pm 1.59 \%$ in glycyrrhizin-treated MCAO rats, $P<0.001$ ) (Fig. $5 \mathrm{c}$ and d). Interestingly, glycyrrhizin inhibits HMGB1 release to levels similar to those we observed in MCAO rats treated with hypothermia. Together, these results suggest that the extracellular release of HMGB1 during an ischemic event is essential in the spread of ischemic injury and that therapeutic hypothermia prevents this spread via inhibition of HMGB1.

\section{Intracerebroventricular injection of HMGB1 neutralizing antibodies prevents ischemic brain injury}

To determine the effect of a more specific inhibition of HMGB1 on ischemic injury, we injected $5 \mu \mathrm{g}$ of a 


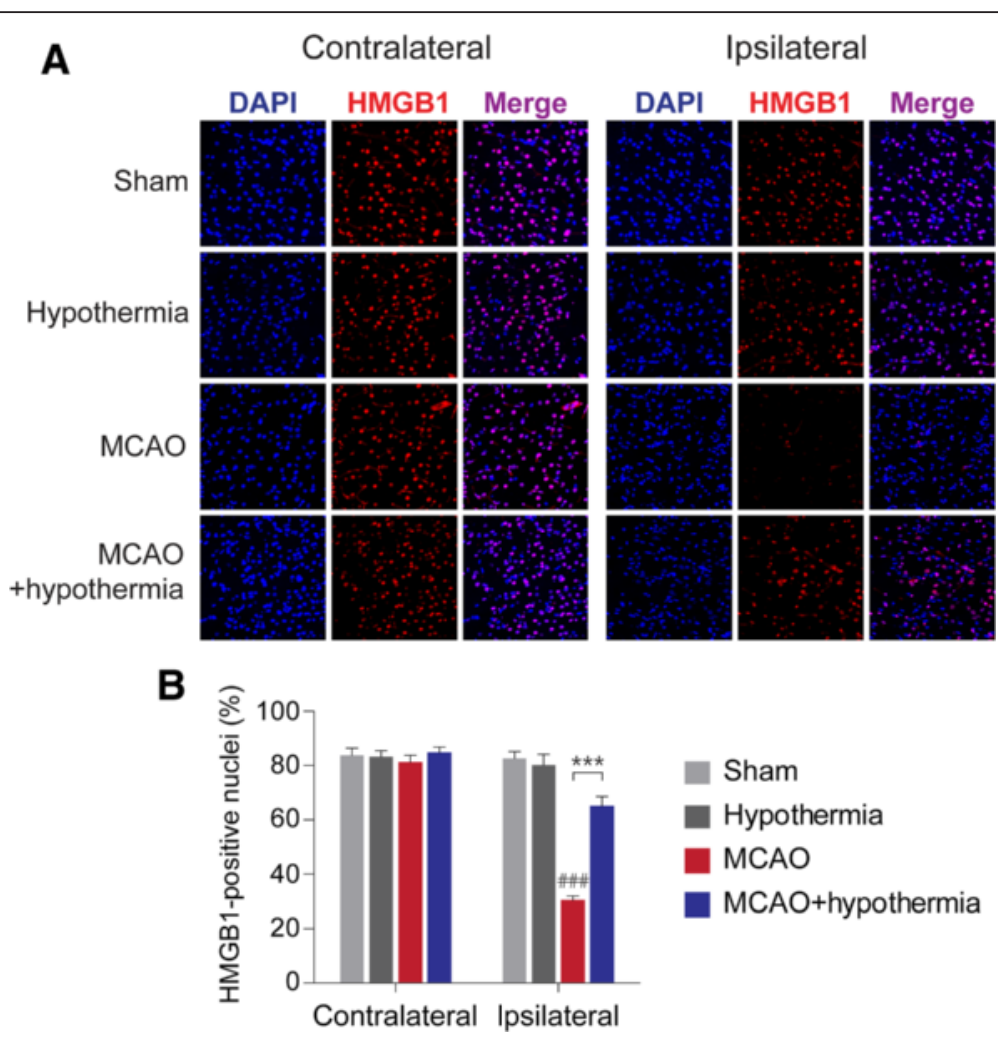

Fig. 2 Hypothermia restores HMGB1 immunoreactivity in post-ischemic MCAO rat brains. a, Representative immunohistochemistry results from MCAO-treated rat brains in the absence or presence of hypothermia. $\mathbf{b}$, A quantification of the immunohistochemistry results in $\mathbf{a}$. The number of rats in each group was as follows: sham $(n=4)$, hypothermia $(n=4)$, MCAO $(n=6)$, MCAO + hypothermia $(n=4)$. \#\#\# $P<0.001$ versus sham alone, *** $P<0.001$ comparing MCAO with and without hypothermia, one-way analysis of variance (ANOVA) followed by the Bonferroni post hoc test

neutralizing antibody against HMGB1 into the intracerebroventricular space of rats. We injected the HMGB1 neutralizing antibody using an infusion pump over the course of $5 \mathrm{~min}$ beginning $30 \mathrm{~min}$ prior to the onset of ischemia. TTC staining after $4 \mathrm{~h}$ of ischemia showed that HMGB1 neutralizing antibody treatment reduces $\mathrm{MCAO}$-induced cortical infarct volume (Fig. 5e and $\mathrm{f}$, see also in Additional file 5: Figure S5). This result provides further evidence for our hypothesis that HMGB1 inhibition effectively protects the brain against the spread of ischemic injury.

\section{Both therapeutic hypothermia and glycyrrhizin inhibit} inflammatory cytokine expression in peri-infarct regions Inflammatory cytokines released from the ischemic penumbra likely contribute to the extensive damage in the penumbra after an acute ischemic stroke [35-38]. To determine whether therapeutic hypothermia and glycyrrhizin alter the expression of inflammatory cytokines, we used RT-PCR to examine the mRNA levels of two major inflammatory cytokines (i.e., IL-1 $\beta$ and TNF- $\alpha$ ) in the peri-infarct region $4 \mathrm{~h}$ after MCAO. We defined the peri-infarct region or penumbra as tissue within $2 \mathrm{~mm}$ of the infarct border. We decided to use the hindlimb region of the primary sensory cortex for our penumbral expression analysis because it consistently fell within the $2 \mathrm{~mm}$ range in all the brain slices we used for TTC staining and because it lies within a region showing perfusion-diffusion mismatch in a permanent MCAO rat model [39]. We found that although ischemic injury increases the expression of interleukin-1 $\beta$ (IL-1 $\beta$ ) in the peri-infarct region, both hypothermia and glycyrrhizin treatments prevent this increase (Fig. 6a). Similarly, MCAO-induced ischemic injury increases peri-infarct tissue necrosis factor- $\alpha$ (TNF- $\alpha$ ) expression, but both hypothermia and glycyrrhizin significantly reduce this increase (Fig. 6b). These results suggest hypothermia helps prevent infarct propagation by suppressing inflammatory cytokine production in peri-infarct regions via its inhibition of HMGB1.

\section{Discussion}

In rodent models of stroke, therapeutic hypothermia reportedly reduces infarct volume and post-ischemic inflammation, salvaging much of the ischemic penumbra $[16,18,19,21,40]$. Previously, Koda et al. reported that hypothermia reduces HMGB1 in rat cortical lysates after bilateral common carotid artery (CCA) occlusion and 


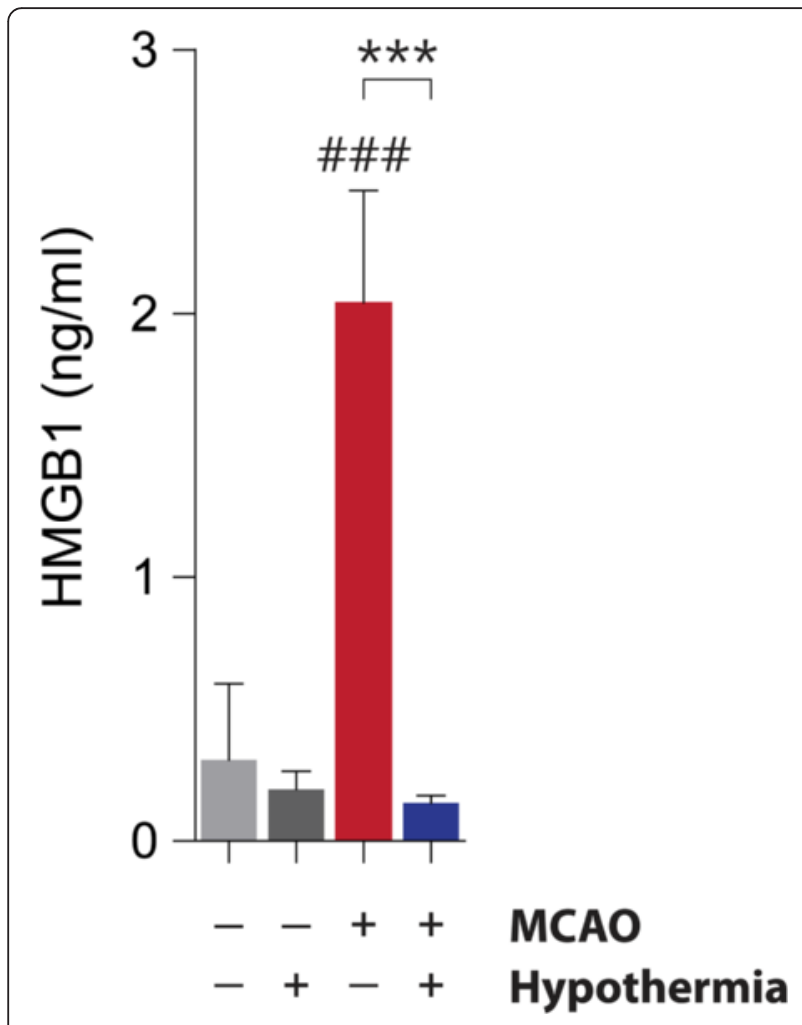

Fig. 3 Hypothermia reduces serum HMGB1 levels in MCAO rats. ELISA assay for HMGB1 performed on sera drawn from rats $4 \mathrm{~h}$ after sham or MCAO surgery in the absence or presence of hypothermia. The number of rats in each group was as follows: sham $(n=4)$, hypothermia $(n=4)$, MCAO $(n=4), \mathrm{MCAO}+$ hypothermia $(n=4)$. \#\#\# $P<0.001$ versus sham alone, ${ }^{* *} P<0.001$ comparing MCAO with and without hypothermia, one-way analysis of variance (ANOVA) followed by the Bonferroni post hoc test

hypotension [41]. Although the ischemic models (i.e., bilateral CCA occlusion with hypotension vs. MCAO) and duration of ischemic injury (i.e., $120 \mathrm{~min}$ reperfusion after $10 \mathrm{~min}$ ischemia vs. $4 \mathrm{~h}$ MCAO in our study) were different, both studies support the intriguing new hypothesis that hypothermia acts to inhibit HMGB1 in acute ischemic injury. This idea is even more clinically relevant in light of a recent study showing that serum HMGB1 levels may be a valuable prognostic marker in stroke patients [31]. It remains unclear, however, whether hypothermia suppresses HMGB1 action at the site of injury, as well as in adjacent and distant tissues in vivo. It is also unclear how HMGB1 participates in ischemic injury propagation and exactly what the consequences of its suppression may be. Our current study is the first direct functional and mechanistic link between HMGB1 and therapeutic hypothermia in a clinically relevant permanent MCAO animal model.

We have also shown that the HMGB1 inhibitor glycyrrhizin attenuates pro-inflammatory cytokines in
MCAO rat brains just like therapeutic hypothermia does. This suggests hypothermia's inhibition of HMGB1 accounts for its suppression of peri-infarct inflammation. IL- $1 \beta$ and TNF- $\alpha$ dramatically affect infarct evolution in experimental stroke models [38, 42, 43]. Indeed, neutralization of IL-1 $\beta$ and TNF- $\alpha$ reduces infarct size in the MCAO model $[44,45]$. Cytokines are produced either in the peri-infarct region or in numerous mini-penumbras inside the infarct core, and both sources likely promote the propagation of ischemic damage $[46,47]$. According to some reports, IL-1 $\beta$ and TNF- $\alpha$ expression begin to increase between 4 and $6 \mathrm{~h}$ after the onset of ischemia $[38,46,48]$. In the permanent MCAO model, though, a substantial fraction of the penumbra is recruited into the infarct core even $1 \mathrm{~h}$ after the onset of ischemia $[38,49]$. This makes it seem unlikely that these cytokines and the HMGB1 that induces them are important in the evolution of a reversible episode of ischemia to an area of irreversible damage. In our real-time PCR experiments, though, we already observed robust increases of both IL-1 $\beta$ and TNF- $\alpha$ expression at $4 \mathrm{~h}$ of permanent MCAO induced ischemia. The fact that other studies generally measure changes in cytokine expression in whole brain lysates rather than a defined periinfarct region $[46,48]$ may hinder their ability to observe early and highly localized mRNA changes. Our new data from the peri-infarct region suggest the involvement of pro-inflammatory cytokines in infarct development and ischemic injury propagation much earlier than previously thought. Hypothermia seems to interfere with these processes via its inhibition of HMGB1. Our results also suggest that HMGB1 inhibitors are worth being assessed for their ability to act as neuroprotectants in human ischemic stroke. Glycyrrhizin has been used to treat chronic hepatitis $C$ infection [50]. However, it necessitates more pre-clinical and clinical studies to prove its efficacy and safety in ischemic stroke patients.

One advantage of hypothermia is that it may protect against ischemic injury even if it is applied after the ischemic insult [17]. Koda et al. reported that hypothermia induced after an ischemic event fails to reduce serum HMGB1 levels in rats [41], suggesting that HMGB1 is irrelevant for hypothermia's protective effects. In contrast, we found that induction of hypothermia $15 \mathrm{~min}$ after an ischemic insult effectively reduces both serum HMGB1 and infarct volume. Consistent with our results, Liu et al. found that post-ischemic administration of a neutralizing antibody against HMGB1 protects against ischemic brain injury [51]. This implies that the effectiveness of post-ischemic hypothermia is indeed related to its inhibition of HMGB1. We expect that further research into the relationships between the time of hypothermia application, the extent of HMGB1 release, and the degree of therapeutic effect will be enormously beneficial. 


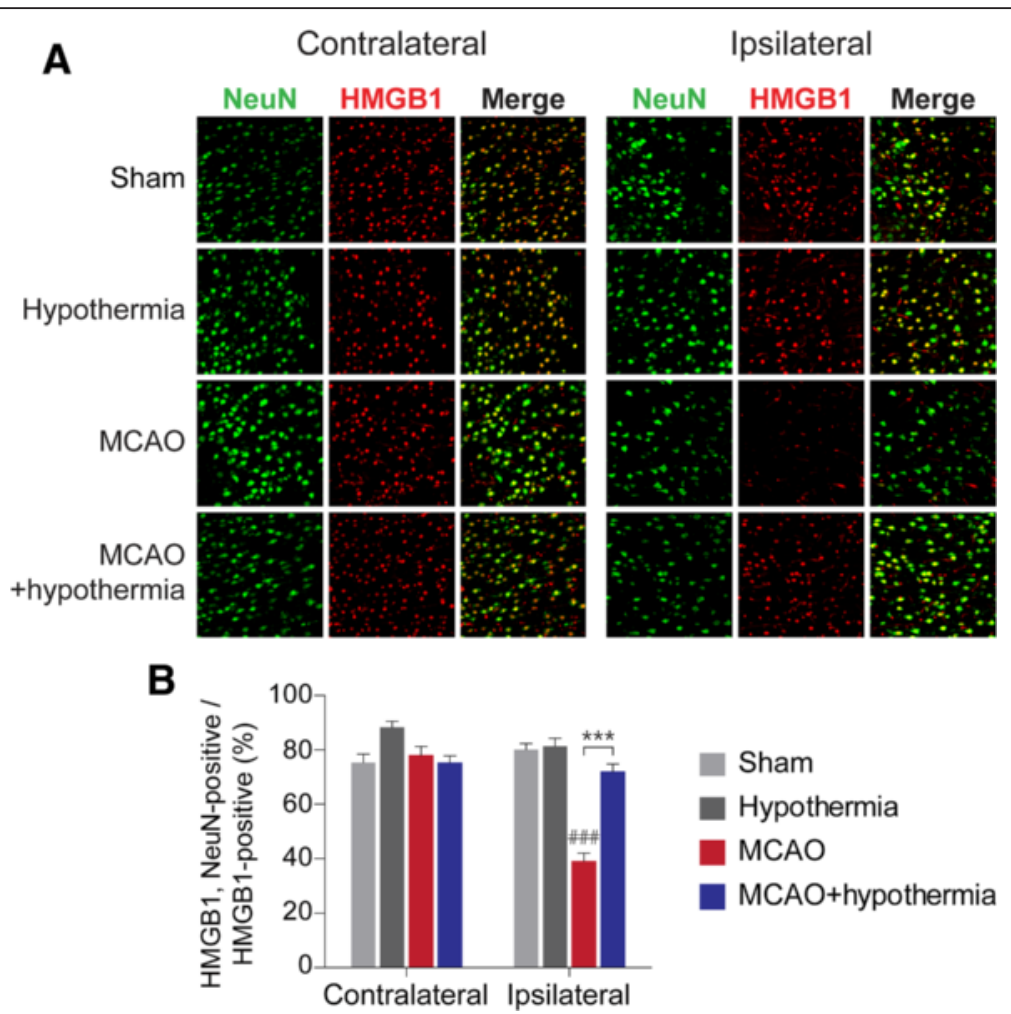

Fig. 4 Hypothermia reverses the decrease in NeuN/HMGB1-double positive cells in MCAO rat brains. a, Representative immunohistochemistry results using antibodies against NeuN and HMGB1. b, A quantification of the immunohistochemistry results in $\mathbf{a}$. The number of rats in each group was as follows: sham $(n=3)$, hypothermia $(n=3)$, MCAO $(n=5)$, MCAO + hypothermia $(n=3)$. \#\#\# $P<0.001$ versus sham alone, *** $P<0.001$ comparing MCAO with and without hypothermia, one-way analysis of variance (ANOVA) followed by the Bonferroni post hoc test

This study provides evidence that therapeutic hypothermia inhibits the propagation of ischemic brain damage by inhibiting the extracellular release of HMGB1.

\section{Methods}

\section{Animal preparation}

Healthy male Wistar rats weighing 295-315 g were used for all experiments. All animal experiments were performed in compliance with guidelines approved by the Institutional Animal Care and Use Committee (IACUC) of Yonsei University Health System and according to National Institutes of Health guidelines.

\section{Experimental MCAO model}

Focal brain ischemia was induced via intraluminal suturing of the middle cerebral artery [52]. Anesthesia was induced with $5 \%$ isoflurane in a mixture of $0.7 \mathrm{~L} / \mathrm{min}$ nitrous oxide and $0.3 \mathrm{~L} / \mathrm{min}$ oxygen and maintained using $2 \%$ isoflurane in the same gas mixture. The external carotid artery (ECA) was ligated and coagulated after isolating it and its branches. The internal carotid artery (ICA) was also carefully isolated from the adjacent vagus nerve. After ligation of the pterygopalatine artery, the common carotid artery (CCA) was also ligated. Next, the proximal ICA was loosely tied with a 6-0 black silk suture, and a microvascular clip was applied across the distal ICA. After making an incision in the proximal ICA, an intraluminal 4-0 MCAO suture (403556PK10, Doccol corporation, Sharon, MA) was inserted and the loosely tied 6-0 black silk suture on the proximal portion of ICA was tightened. After removing the clip, an intraluminal 4-0 MCAO suture was advanced from the proximal ICA lumen in the distal direction to a point approximately $22 \mathrm{~mm}$ beyond the CCA bifurcation. For drug treatment, glycyrrhizin $(100 \mathrm{mg} / \mathrm{kg})$ was injected intraperitoneally $30 \mathrm{~min}$ before the onset of MCAO.

\section{Temperature management}

Animals were randomly divided into four groups: sham, hypothermia, MCAO, and MCAO + hypothermia. In the hypothermia group, surface cooling was begun $15 \mathrm{~min}$ after ischemic induction by placing ice packs on the rat torso. Vecuronium $(0.9 \mathrm{mg} / \mathrm{kg})$ was injected intramuscularly to inhibit shivering. After the sham and MCAO surgeries, the target core temperatures were carefully monitored and maintained for $4 \mathrm{~h}$ using a feedbackcontrolled heating pad (HB 101, Harvard apparatus, Holliston, MA) and surface cooling with ice packs. 

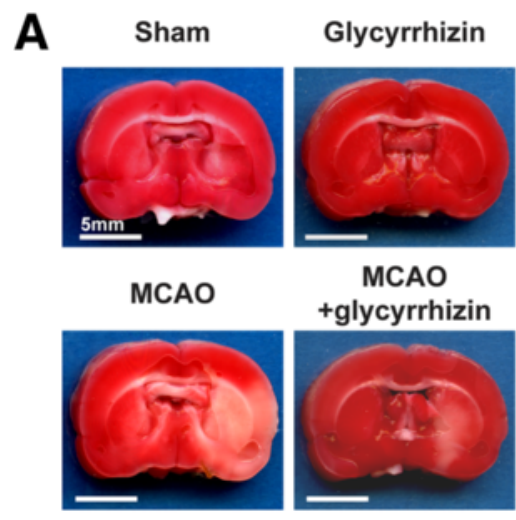

MCAO +glycyrrhizin

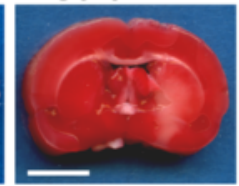

C

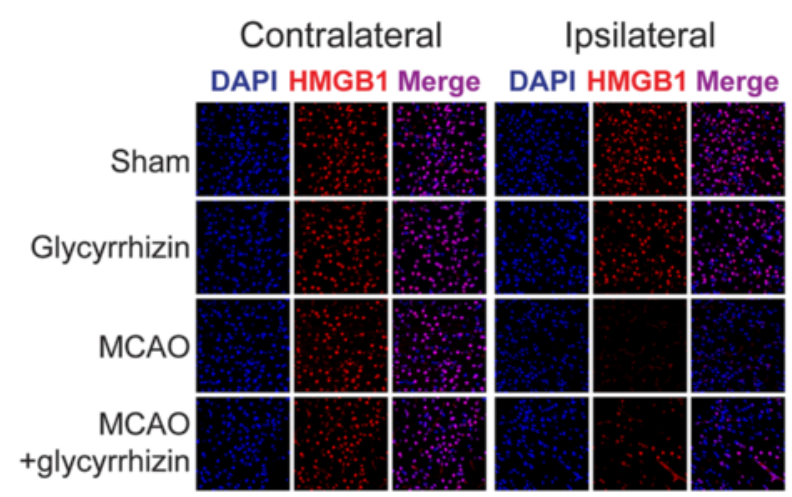

D
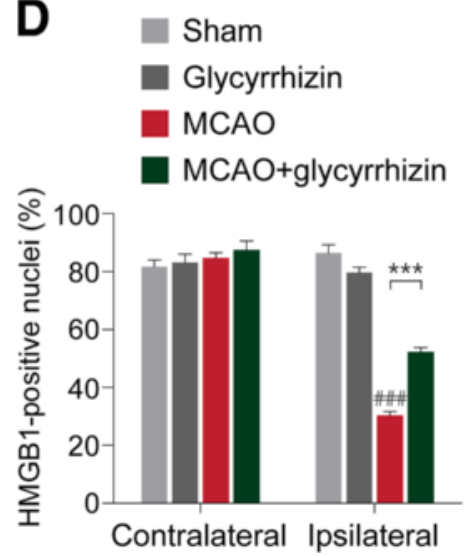

E
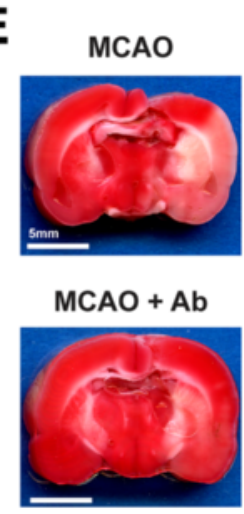

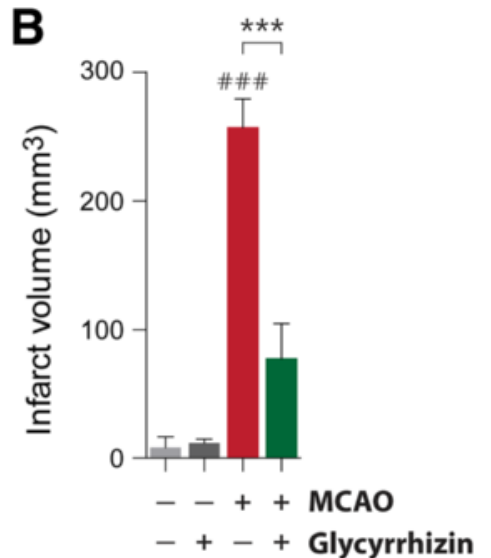

-++ Glycyrrhizin

Fig. 5 Glycyrrhizin and HMGB1 neutralizing antibodies reduce MCAO-induced ischemic brain injury. a, Representative $T T C$-staining results. A mixture of glycyrrhizin $(100 \mathrm{mg} / \mathrm{kg})$ and saline was administered intraperitoneally $30 \mathrm{~min}$ before MCAO. $\mathbf{b}, \mathrm{A}$ quantification of the TTC staining results in a. The number of rats in each group was as follows: sham $(n=4)$, glycyrrhizin $(n=4), \operatorname{MCAO}(n=5)$, MCAO + glycyrrhizin $(n=5)$. \#\#\# $P<0.001$ versus sham alone, *** $P<0.001$ comparing MCAO with and without glycyrrhizin, one-way analysis of variance (ANOVA) followed by the Bonferroni post hoc test. $\mathbf{c}$, Representative images showing HMGB1 immunoreactivity in brain sections from MCAO and/or glycyrrhizin-treated rats. $\mathbf{d}$, A quantification of the immunohistochemistry results in $\mathbf{c}$. The number of rats in each group was as follows: sham $(n=3)$, glycyrrhizin $(n=3)$, MCAO $(n=4)$, MCAO + glycyrrhizin $(n=4)$. \#\#\# $P<0.001$ versus sham alone, *** $P<0.001$ comparing MCAO with and without glycyrrhizin, one-way ANOVA followed by the Bonferroni post hoc test. e. Representative images of TTC staining results. HMGB1 neutralizing antibodies ( $5 \mu \mathrm{g}$ of antibody in $5 \mu \mathrm{l}$ of PBS) were administered to the rats by intracerebroventricular injection 30 min before MCAO. $\mathbf{f}$, A quantification of the TTC staining results in e. The number of rats in each group was as follows: MCAO $(n=3), M C A O+$ neutralizing antibody $(n=8)$. ${ }^{* *} P<0.01$ comparing MCAO with and without the neutralizing antibody, unpaired $t$-test 


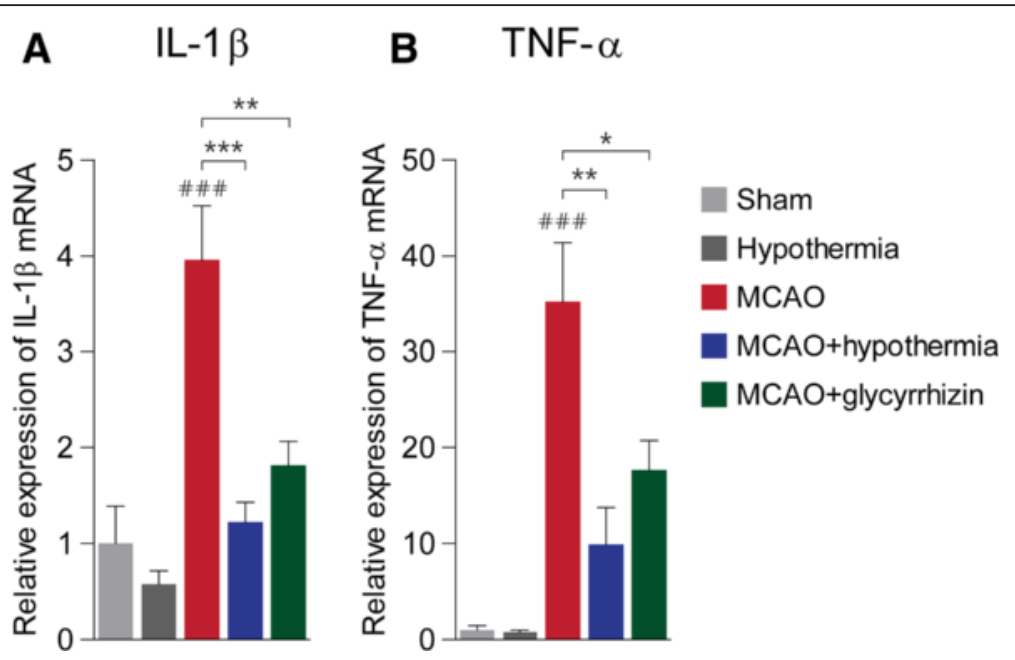

Fig. 6 Pro-inflammatory cytokine expression in the peri-infarct region after MCAO. a, Quantification of interleukin-1 $\beta$ (IL-1 $\beta$ ) expression by RT-PCR. \#\#\# $P<0.001$ versus sham alone, ${ }^{* *} P<0.01,{ }^{* * *} P<0.001$, one-way analysis of variance (ANOVA) followed by the Bonferroni post hoc test. b. Quantification of tissue necrosis factor-a (TNF-a) expression by RT-PCR. \#\#\# $P<0.001$ versus sham alone, ${ }^{*} P<0.05$, ** $P<0.01$, one-way ANOVA followed by the Bonferroni post hoc test. The number of rats in each group was as follows: sham $(n=5)$, hypothermia $(n=5), \operatorname{MCAO}(n=8)$, $\mathrm{MCAO}+$ hypothermia $(n=6), \mathrm{MCAO}+$ glycyrrhizin $(n=9)$

\section{Infarct volume measurement}

Rats were decapitated under anesthesia $4 \mathrm{~h}$ after sham or MCAO surgery. Coronal sections ( $2 \mathrm{~mm}$-thick) were stained with $1 \%$ TTC (T8877, Sigma-Aldrich, St. Louis, MO) solution at $37{ }^{\circ} \mathrm{C}$ for $10 \mathrm{~min}$. After fixation, caudal and rostral faces of each section was scanned with a flatbed scanner. Scanned images were analyzed with ImageJ $1.48 \mathrm{v}$ (NIH, Bethesda, MD). Infarcted volumes $\left(\mathrm{mm}^{3}\right)$ were calculated by multiplying the total averaged infarcted area by the section thickness; Thickness $\mathrm{x}$ (caudal area + rostral area) $/ 2$.

\section{Enzyme-linked immunosorbent assay (ELISA)}

One $\mathrm{ml}$ of blood from the right atrium was withdrawn using a 23-gauge needle into a serum separator tube (BD, Plymouth, UK), centrifuged for $20 \mathrm{~min}$ at 2,000 rpm. HMGB1 concentrations were determined using the HMGB1 ELISA kit (ST51011, IBL International GmbH, Hamburg, Germany).

\section{Immunofluorescence}

Two mm-thick rat brain slices (bregma $0.7 \mathrm{~mm}$ to $-1.3 \mathrm{~mm}$ ) were immersed in a $4 \%$ paraformaldehyde solution and then cryoprotected with $30 \%$ sucrose in phosphate-buffered saline (PBS). Sections with a thickness of $20 \mu \mathrm{m}$ from $0.2 \mathrm{~mm}$ to $-0.3 \mathrm{~mm}$ relative to the bregma were chosen for staining. Sections were first permeabilized and then incubated overnight at $4{ }^{\circ} \mathrm{C}$ with an anti-HMGB1 antibody (1:100, ab18256; Abcam, Cambridge, UK) or an anti-NeuN antibody (1:100, MAB377; Millipore, Billerica, MA). After washing, fluorescent dye-conjugated secondary antibodies were applied. Stained sections were observed under a LSM700 confocal microscope (Carl Zeiss, Jena, Germany).

\section{MAP-2 (microtubule associated protein-2) staining}

Two mm-thick brain slices (bregma $-1.3 \mathrm{~mm}$ to $-3.3 \mathrm{~mm}$ ) were immersed in a $4 \%$ paraformaldehyde solution and then embedded in paraffin. Paraffin sectioning (4 $\mu \mathrm{m}$-thick) was performed for immunohistochemistry. After deparaffinization, sections were incubated with an anti-MAP-2 antibody (1:100, M1406, Sigma) at $4{ }^{\circ} \mathrm{C}$. After incubation with HRP-conjugated secondary antibodies, sections were stained with diaminobenzidine (K-3468, Dako). Images of sections were obtained using a motorized microscope (BX61VS, Olympus).

\section{Neurobehavioral testing}

Hypothermic rats were passively rewarmed to $37{ }^{\circ} \mathrm{C}$ for $1 \mathrm{~h}$ after $3 \mathrm{~h} \mathrm{MCAO}$ and were subjected to neurobehavioral tests. A modified Garcia 18-point scoring system was used for the evaluation of neurological deficits [53]. Six sensorimotor tests (spontaneous activity, symmetry in the movement of four limbs, forepaw outstretching, climbing, body proprioception, response to vibrissae touch; each scored on a scale of $0 \sim 3$ or $1 \sim 3$ ) were performed and the scores given to each rat were summated to derive total neurological deficit score (the maximum score 18, namely, healthy rats and the minimum score 3 ).

\section{Real-time polymerase chain reaction (RT-PCR)}

Tissue RNA was extracted using the Hybrid-R kit (305-010, GeneAll biotechnology, Seoul, Korea). cDNAs were prepared from $1 \mu \mathrm{g}$ of total RNA using 
the PrimeScript 1st strand cDNA Synthesis Kit (Takara Bio, Shiga, Japan). PCR amplification was performed using the SYBR-Green reagent (Takara Bio).

\section{Statistical analysis}

All data are presented as means \pm s.e.m. Differences between groups were analyzed using two-tailed unpaired $t$ tests or one-way analysis of variance (ANOVAs) followed by Bonferroni post hoc tests for multiple comparisons between groups. $P<0.05$ was considered significant.

\section{Additional files}

Additional file 1: Figure S1. Representative image of TTC-stained serial coronal brain sections from MCAO-treated rats. (DOCX 1229 kb)

Additional file 2: Figure S2. Comparison of infarct volume between TTC- and MAP-2-stained coronal brain sections. (DOCX $872 \mathrm{~kb}$ )

Additional file 3: Figure S3. Neurobehavioral tests that assess MCAO-induced functional neurological deficits. (DOCX $34 \mathrm{~kb}$ )

Additional file 4: Figure S4. Representative image of $\Pi$ T-stained serial coronal brain sections from MCAO and/or glycyrrhizin-treated rats. (DOCX $1213 \mathrm{~kb}$ )

Additional file 5: Figure S5. Representative image of TTC-stained serial coronal brain sections from MCAO and/or anti-HMGB1 antibodies-treated rats. (DOCX $1204 \mathrm{~kb}$ )

\section{Acknowledgements}

Not applicable.

\section{Funding}

J.S. You was supported by the Basic Science Research Program of the National Research Foundation of Korea (NRF) funded by the Ministry of Education (NRF-2013R1A1A2009391) and by the Ministry of Science, ICT \& Future Planning (NRF-2015R1C1A1A01054641), a faculty research grant of Yonsei University College of Medicine for 2014 (6-2014-0059), and the Yonsei University Future-leading Research Initiative for 2015 (2015-22-0096). C.H. Kim was supported by the Brain Research Program through the National Research Foundation of Korea (NRF) funded by the Ministry of Science, ICT \& Future Planning (NRF-2015M3C7A1028396 \& 2016M3C7A1913844) \& by a National Research Foundation of Korea (NRF) grant from the Korea government (MSIP) (NRF-2014R1A2A1A11051372). The funding bodies had no role in the design, collection, analysis or interpretation of this study.

\section{Availability of data and material}

All data and materials are available upon requests.

\section{Authors' contribution}

CHK, JSY, JHL designed and planned the study. JSY, JHL, EJY, JS performed experiments and generated results. JSY, JHL, EJY, AK, YEC, SPC, IP analyzed data. CHK and JSY interpreted the results. YEC, SPC, IP contributed the reagents and assisted in designing the study. CHK, JSY, JHL, AK wrote and edited the manuscript. All authors read and approved the final manuscript.

\section{Competing interests}

The authors declare that they have no competing interests.

\section{Consent for publication}

Not applicable.

\section{Ethics approval and consent to participate}

All animal experiments were performed in compliance with guidelines approved by the Institutional Animal Careand Use Committee (IACUC) of Yonsei University Health System (reference number: 2014-0139).

\section{Author details}

${ }^{1}$ Department of Pharmacology, BK21 PLUS Project for Medical Science, Brain Research Institute, Yonsei University College of Medicine, 50-1 Yonsei-ro, Seodaemun-gu, Seoul 03722, South Korea. ${ }^{2}$ Department of Radiology, Yonsei University College of Medicine, Seoul 03722, South Korea. ${ }^{3}$ Department of Emergency Medicine, Yonsei University College of Medicine, 50-1 Yonsei-ro, Seodaemun-gu, Seoul 03722, South Korea. ${ }^{4}$ Severance Biomedical Science Institute, Yonsei University College of Medicine, Seoul 03722, South Korea.

Received: 5 April 2016 Accepted: 10 August 2016

Published online: 20 August 2016

\section{References}

1. Chen ZM, Sandercock P, Pan HC, Counsell C, Collins R, Liu LS, et al. Indications for early aspirin use in acute ischemic stroke : A combined analysis of 40000 randomized patients from the chinese acute stroke trial and the international stroke trial. On behalf of the CAST and IST collaborative groups. Stroke. 2000;31(6):1240-9.

2. Smith WS, Sung G, Saver J, Budzik R, Duckwiler G, Liebeskind DS, et al. Mechanical thrombectomy for acute ischemic stroke: final results of the Multi MERCI trial. Stroke. 2008;39(4):1205-12.

3. Tissue plasminogen activator for acute ischemic stroke. The National Institute of Neurological Disorders and Stroke rt-PA Stroke Study Group. N Engl J Med. 1995;333(24):1581-7.

4. Prabhakaran S, Ruff I, Bernstein RA. Acute stroke intervention: a systematic review. JAMA. 2015;313(14):1451-62.

5. Hacke W, Kaste M, Bluhmki E, Brozman M, Davalos A, Guidetti D, et al. Thrombolysis with alteplase 3 to 4.5 hours after acute ischemic stroke. N Engl J Med. 2008;359(13):1317-29.

6. Lacy CR, Suh DC, Bueno M, Kostis JB. Delay in presentation and evaluation for acute stroke: Stroke Time Registry for Outcomes Knowledge and Epidemiology (S.T.R.O.K.E.). Stroke. 2001;32(1):63-9.

7. Nedeltchev K, Arnold M, Brekenfeld C, Isenegger J, Remonda L, Schroth G, et al. Pre- and in-hospital delays from stroke onset to intra-arterial thrombolysis. Stroke. 2003:34(5):1230-4

8. Astrup J, Siesjo BK, Symon L. Thresholds in cerebral ischemia - the ischemic penumbra. Stroke. 1981;12(6):723-5.

9. Heiss WD. Ischemic penumbra: evidence from functional imaging in man. J Cereb Blood Flow Metab. 2000;20(9):1276-93.

10. Parsons MW, Barber PA, Chalk J, Darby DG, Rose S, Desmond PM, et al. Diffusion- and perfusion-weighted MRI response to thrombolysis in stroke. Ann Neurol. 2002;51(1):28-37.

11. Donnan GA, Davis SM, Parsons MW, Ma H, Dewey HM, Howells DW. How to make better use of thrombolytic therapy in acute ischemic stroke. Nat Rev Neurol. 2011;7(7):400-9.

12. Yenari MA, Hemmen TM. Therapeutic hypothermia for brain ischemia: where have we come and where do we go? Stroke. 2010;41(10 Suppl):S72-4.

13. Bernard SA, Gray TW, Buist MD, Jones BM, Silvester W, Gutteridge G, et al. Treatment of comatose survivors of out-of-hospital cardiac arrest with induced hypothermia. N Engl J Med. 2002;346(8):557-63.

14. De Georgia MA, Krieger DW, Abou-Chebl A, Devlin TG, Jauss M, Davis SM, et al. Cooling for Acute Ischemic Brain Damage (COOL AID): a feasibility trial of endovascular cooling. Neurology. 2004;63(2):312-7.

15. Hemmen TM, Raman R, Guluma KZ, Meyer BC, Gomes JA, Cruz-Flores S, et al. Intravenous thrombolysis plus hypothermia for acute treatment of ischemic stroke (ICTUS-L): final results. Stroke. 2010;41(10):2265-70.

16. Chen H, Chopp M, Zhang ZG, Garcia JH. The effect of hypothermia on transient middle cerebral artery occlusion in the rat. J Cereb Blood Flow Metab. 1992;12(4):621-8.

17. Colbourne F, Corbett D, Zhao Z, Yang J, Buchan AM. Prolonged but delayed postischemic hypothermia: a long-term outcome study in the rat middle cerebral artery occlusion model. J Cereb Blood Flow Metab. 2000;20(12): 1702-8.

18. Yanamoto H, Nagata I, Niitsu Y, Zhang Z, Xue JH, Sakai N, et al. Prolonged mild hypothermia therapy protects the brain against permanent focal ischemia. Stroke. 2001;32(1):232-9.

19. Han HS, Qiao Y, Karabiyikoglu M, Giffard RG, Yenari MA. Influence of mild hypothermia on inducible nitric oxide synthase expression and reactive nitrogen production in experimental stroke and inflammation. J Neurosci. 2002;22(10):3921-8. 
20. Busto R, Globus MY, Dietrich WD, Martinez E, Valdes I, Ginsberg MD. Effect of mild hypothermia on ischemia-induced release of neurotransmitters and free fatty acids in rat brain. Stroke. 1989;20(7):904-10.

21. Deng H, Han HS, Cheng D, Sun GH, Yenari MA. Mild hypothermia inhibits inflammation after experimental stroke and brain inflammation. Stroke. 2003;34(10):2495-501.

22. MacLellan CL, Davies LM, Fingas MS, Colbourne F. The influence of hypothermia on outcome after intracerebral hemorrhage in rats. Stroke. 2006;37(5):1266-70.

23. ladecola C, Anrather J. The immunology of stroke: from mechanisms to translation. Nat Med. 2011;17(7):796-808.

24. Kim JB, Sig Choi J, Yu YM, Nam K, Piao CS, Kim SW, et al. HMGB1, a novel cytokine-like mediator linking acute neuronal death and delayed neuroinflammation in the postischemic brain. J Neurosci. 2006;26(24):6413-21.

25. Andersson U, Tracey KJ. HMGB1 is a therapeutic target for sterile inflammation and infection. Annu Rev Immunol. 2011;29:139-62.

26. Muhammad S, Barakat W, Stoyanov S, Murikinati S, Yang H, Tracey KJ, et al The HMGB1 receptor RAGE mediates ischemic brain damage. J Neurosci. 2008;28(46):12023-31.

27. Hori O, Brett J, Slattery T, Cao R, Zhang J, Chen JX, et al. The receptor for advanced glycation end products (RAGE) is a cellular binding site for amphoterin. Mediation of neurite outgrowth and co-expression of rage and amphoterin in the developing nervous system. J Biol Chem. 1995;270(43): 25752-61.

28. Park JS, Svetkauskaite D, He Q, Kim JY, Strassheim D, Ishizaka A, et al. Involvement of toll-like receptors 2 and 4 in cellular activation by high mobility group box 1 protein. J Biol Chem. 2004;279(9):7370-7.

29. Qiu J, Nishimura M, Wang Y, Sims JR, Qiu S, Savitz SI, et al. Early release of HMGB-1 from neurons after the onset of brain ischemia. J Cereb Blood Flow Metab. 2008;28(5):927-38.

30. Qiu J, Xu J, Zheng Y, Wei Y, Zhu X, Lo EH, et al. High-mobility group box 1 promotes metalloproteinase-9 upregulation through Toll-like receptor 4 after cerebral ischemia. Stroke. 2010;41(9):2077-82.

31. Huang JM, Hu J, Chen N, Hu ML. Relationship between plasma highmobility group box-1 levels and clinical outcomes of ischemic stroke. J Crit Care. 2013;28(5):792-7.

32. Mollica L, De Marchis F, Spitaleri A, Dallacosta C, Pennacchini D, Zamai M, et al. Glycyrrhizin binds to high-mobility group box 1 protein and inhibits its cytokine activities. Chem Biol. 2007;14(4):431-41.

33. Gong G, Xiang L, Yuan L, Hu L, Wu W, Cai L, et al. Protective effect of glycyrrhizin, a direct HMGB1 inhibitor, on focal cerebral ischemia/ reperfusion-induced inflammation, oxidative stress, and apoptosis in rats. PLoS One. 2014;9(3):e89450.

34. Kim SW, Jin Y, Shin JH, Kim ID, Lee HK, Park S, et al. Glycyrrhizic acid affords robust neuroprotection in the postischemic brain via anti-inflammatory effect by inhibiting HMGB1 phosphorylation and secretion. Neurobiol Dis. 2012;46(1):147-56.

35. Davies CA, Loddick SA, Toulmond S, Stroemer RP, Hunt J, Rothwell NJ. The progression and topographic distribution of interleukin-1beta expression after permanent middle cerebral artery occlusion in the rat. J Cereb Blood Flow Metab. 1999;19(1):87-98.

36. Lambertsen KL, Gregersen R, Finsen B. Microglial-macrophage synthesis of tumor necrosis factor after focal cerebral ischemia in mice is strain dependent. J Cereb Blood Flow Metab. 2002;22(7):785-97.

37. Luheshi NM, Kovacs KJ, Lopez-Castejon G, Brough D, Denes A. Interleukin1alpha expression precedes IL-1beta after ischemic brain injury and is localised to areas of focal neuronal loss and penumbral tissues. J Neuroinflammation. 2011;8:186.

38. Lambertsen KL, Biber K, Finsen B. Inflammatory cytokines in experimental and human stroke. J Cereb Blood Flow Metab. 2012;32(9):1677-98.

39. Reid E, Graham D, Lopez-Gonzalez MR, Holmes WM, Macrae IM, McCabe C. Penumbra detection using PWI/DWI mismatch MRI in a rat stroke model with and without comorbidity: comparison of methods. J Cereb Blood Flow Metab. 2012;32(9):1765-77.

40. Choi KE, Hall CL, Sun JM, Wei L, Mohamad O, Dix TA, et al. A novel stroke therapy of pharmacologically induced hypothermia after focal cerebral ischemia in mice. FASEB J. 2012;26(7):2799-810.

41. Koda Y, Tsuruta R, Fujita M, Miyauchi T, Kaneda K, Todani M, et al. Moderate hypothermia suppresses jugular venous superoxide anion radical, oxidative stress, early inflammation, and endothelial injury in forebrain ischemia/ reperfusion rats. Brain Res. 2010;1311:197-205.
42. Allan SM, Tyrrell PJ, Rothwell NJ. Interleukin-1 and neuronal injury. Nat Rev Immunol. 2005:5(8):629-40.

43. Hallenbeck JM. The many faces of tumor necrosis factor in stroke. Nat Med. 2002;8(12):1363-8.

44. Yamasaki Y, Matsuura N, Shozuhara H, Onodera H, Itoyama Y, Kogure K. Interleukin-1 as a pathogenetic mediator of ischemic brain damage in rats. Stroke. 1995;26(4):676-80.

45. Barone FC, Arvin B, White RF, Miller A, Webb CL, Willette RN, et al. Tumor necrosis factor-alpha. A mediator of focal ischemic brain injury. Stroke. 1997;28(6):1233-44.

46. Clausen BH, Lambertsen KL, Meldgaard M, Finsen B. A quantitative in situ hybridization and polymerase chain reaction study of microglialmacrophage expression of interleukin-1 beta mRNA following permanent middle cerebral artery occlusion in mice. Neuroscience. 2005;132(4):879-92.

47. del Zoppo GJ, Sharp FR, Heiss WD, Albers GW. Heterogeneity in the penumbra. J Cereb Blood Flow Metab. 2011;31(9):1836-51.

48. Lambertsen KL, Clausen BH, Babcock AA, Gregersen R, Fenger C, Nielsen HH, et al. Microglia protect neurons against ischemia by synthesis of tumor necrosis factor. J Neurosci. 2009;29(5):1319-30.

49. Hossmann KA. The two pathophysiologies of focal brain ischemia: implications for translational stroke research. J Cereb Blood Flow Metab. 2012;32(7):1310-6.

50. van Rossum TG, Vulto AG, de Man RA, Brouwer JT, Schalm SW. Review article: glycyrrhizin as a potential treatment for chronic hepatitis C. Aliment Pharmacol Ther. 1998;12(3):199-205.

51. Liu K, Mori S, Takahashi HK, Tomono Y, Wake H, Kanke T, et al. Anti-high mobility group box 1 monoclonal antibody ameliorates brain infarction induced by transient ischemia in rats. FASEB J. 2007;21(14):3904-16.

52. Belayev L, Alonso OF, Busto R, Zhao W, Ginsberg MD. Middle cerebral artery occlusion in the rat by intraluminal suture. Neurological and pathological evaluation of an improved model. Stroke. 1996;27(9):1616-22.

53. Garcia JH, Wagner S, Liu K-F, Hu XJ. Neurological Deficit and Extent of Neuronal Necrosis Attributable to Middle Cerebral Artery Occlusion in Rats: Statistical Validation. Stroke. 1995;26(4):627-35.

\section{Submit your next manuscript to BioMed Central and we will help you at every step:}

- We accept pre-submission inquiries

- Our selector tool helps you to find the most relevant journal

- We provide round the clock customer support

- Convenient online submission

- Thorough peer review

- Inclusion in PubMed and all major indexing services

- Maximum visibility for your research

Submit your manuscript at www.biomedcentral.com/submit
C Biomed Central 